\title{
Clinical Use of a Diagnostic Gene Expression Signature for Melanocytic Neoplasms
}

\author{
Jaime Tschen, MD; Paige Davies, MSc; Stephanie M. Meek, PhD; Loren E. Clarke, MD
}

\section{PRACTICE POINT}

- Implementation of a gene expression signature in the diagnosis of histopathologically ambiguous lesions can safely increase diagnostic accuracy and optimize treatment.

A gene expression signature has been validated as an adjunct to traditional methods of differentiating malignant and benign melanocytic neoplasms, and its use in clinical practice warrants further study. This study followed patients whose melanocytic neoplasms were managed according to a benign result from the gene expression signature $(\mathrm{N}=25)$. Eligible patients whose tested lesions were classified as benign by the gene expression signature and were subsequently treated as benign by their dermatology providers were observed for a mean follow-up period of 38.5 months. Results suggest that many patients with melanocytic neoplasms classified as benign by the gene expression signature may safely forego additional surgical excision.

Cutis. 2021;107:264-269.

A ccording to National Institutes of Health estimates, more than 90,000 new cases of melanoma were diagnosed in $2018 .{ }^{1}$ Overall 5-year survival for patients with melanoma exceeds $90 \%$, but individual survival estimates are highly dependent on stage at diagnosis, and survival decreases markedly with metastasis. Therefore, early and accurate diagnosis is critical.

Diagnosis of melanocytic neoplasms usually is performed by dermatopathologists through microscopic examination of stained tissue biopsy sections, a technically simple and effective method that enables a definitive diagnosis of benign nevus or malignant melanoma to be made in most cases. However, approximately $15 \%$ of all biopsied melanocytic lesions will exhibit some degree of histopathologic ambiguity, ${ }^{2-4}$ meaning that some of their microscopic features will be characteristic of a benign nevus while others will suggest the possibility of malignant melanoma. Diagnostic interpretations often vary in these cases, even among experts, and a definitive diagnosis of benign or malignant may be difficult to achieve by microscopy alone..$^{2-4}$ Because of the marked reduction in survival once a melanoma has metastasized, these diagnostically ambiguous lesions often are treated as possible malignant melanomas with complete surgical excision (or re-excision). However, some experts suggest that many histopathologically ambiguous melanocytic neoplasms are, in fact, benign, ${ }^{5}$ a notion supported by epidemiologic evidence. ${ }^{6,7}$ Therefore, excision of many ambiguous melanocytic neoplasms might be avoided if definitive diagnosis could be achieved.

A gene expression signature was developed and validated for use as an adjunct to traditional methods of differentiating malignant melanocytic neoplasms from their benign counterparts. ${ }^{8-11}$ This test quantifies the RNA

Dr. Tschen is from St. Joseph Dermatopathology, Houston, Texas. Ms. Davies is from Assurex Health Ltd, Toronto, Ontario, Canada. Drs. Meek and Clarke are from Myriad Genetics, Inc, Salt Lake City, Utah.

Dr. Tschen reports no conflict of interest. Ms. Davies was employed by Assurex Health Ltd, a Myriad Genetics subsidiary, and Drs. Meek and Clarke were employed by Myriad Genetics, Inc, at the time of manuscript submission.

This study was funded by Myriad Genetics, Inc.

The eTable is available in the Appendix online at www.mdedge.com/dermatology.

Correspondence: Loren E. Clarke, MD, Myriad Genetics, Inc, 320 Wakara Way, Salt Lake City, UT 84108 (Iclarke@myriad.com). doi: 10.12788 /cutis. 0254 
transcripts produced by 14 genes known to be overexpressed in malignant melanomas by comparison to benign nevi. These values are then combined algorithmically with measurements of 9 reference genes to produce an objective numerical score that is classified as benign, malignant, or indeterminate. When used by boardcertified dermatopathologists and dermatologists confronting ambiguous melanocytic lesions, the test produces substantial increases in definitive diagnoses and prompts changes in treatment recommendations. ${ }^{12,13}$ However, the long-term consequences of foregoing surgical excision of melanocytic neoplasms that are diagnostically ambiguous but classified as benign by this test have not yet been formally assessed. In the current study, prospectively tested patients whose ambiguous melanocytic neoplasms were classified as benign by the gene expression signature were followed for up to 4.5 years to evaluate the long-term safety of treatment decisions aligned with benign test results.

\section{Methods}

Study Population-As part of a prior study, ${ }^{12}$ US-based dermatopathologists submitted tissue sections from biopsied melanocytic neoplasms determined to be diagnostically ambiguous by histopathology for analysis with the gene expression signature (Myriad Genetics, Inc). Diagnostically ambiguous lesions were those lesions that were described as ambiguous, uncertain, equivocal, indeterminate, or other synonymous terms by the submitting dermatopathologist and therefore lacked a confident diagnosis of benign or malignant prior to testing. Patients initially were tested between May 2014 and August 2014, with samples submitted through a prospective clinical experience study designed to assess the impact of the test on diagnosis and treatment decisions. This study was performed under an institutional review board waiver of consent (Quorum \#33403/1)

Patients were eligible for inclusion in the current study if their biopsy specimens (1) had an uncertain preliminary diagnosis according to the submitting dermatopathologist (pretest diagnosis of indeterminate); (2) received a negative (benign) score from the gene expression test; (3) were treated as benign by the dermatologist(s) involved in follow-up care; and (4) were submitted by a single site (St. Joseph Medical Center, Houston, Texas). Although a single dermatopathology site was used for this study, multiple dermatologists were involved in the final treatment of these patients. Patients with benign scores who received additional intervention were excluded, as they may have a lower rate of adverse events (ie, metastasis) than those who did not receive intervention and would therefore skew the analysis population. A total of 25 patients from the prior study met these inclusion criteria. The previously collected ${ }^{12}$ pretest and posttest de-identified data were compiled from the commercial laboratory databases, and the patients were followed from the time of testing via medical record review performed by the dermatology providers at participating sites. Clinical follow-up data were collected using studyspecific case report forms (CRFs) that captured the following: (1) the dates and results of clinical followup visits; (2) the type(s) of treatment and interventions (if any) performed at those visits; (3) the specific indication for any intervention performed; (4) any evidence of persistent, locally recurrent, and/or distant melanocytic neoplasia (whether definitively attributable to the tested lesion or not); and (5) death from any cause. The CRF assigned interventions to 1 of 5 categories: excision, excision with sentinel lymph node biopsy, referral to dermatologic or other surgeon, examination only (without surgical intervention), and other. Selection of other required a free-text description of the treatment and indications. Pertinent information not otherwise captured by the CRF also was recordable as free text.

Gene Expression Testing-Gene expression testing was carried out at the time of specimen submission in the prior study ${ }^{12}$ as described previously. ${ }^{14}$ Briefly, formalinfixed, paraffin-embedded, unstained tissue sections and/ or tissue blocks were submitted for testing along with a single hematoxylin and eosin-stained slide used to identify and designate the representative portion(s) of the lesion to be tested. These areas were macrodissected from unstained tissue sections and pooled for RNA extraction. Expression of 14 biomarker genes and 9 reference genes was measured via quantitative reverse transcription-polymerase chain reaction, performed in triplicate for each individual gene. The assay score was generated through application of a weighted algorithm to the expression values generated through quantitative reverse transcription-polymerase chain reaction. Scores were plotted on a scale ranging from -16.7 to 11.1 , with scores from 0.0 to 11.1 classified as malignant, scores from -16.7 to -2.1 as benign, and scores from -2.1 to -0.1 as indeterminate.

Statistical Analysis-Demographic and other baseline characteristics of the patient population were summarized. Follow-up time was calculated as the interval between the date a patient's gene expression test result was first issued to the provider and the date of the patient's last recorded visit during the study period. All patient dermatology office visits within the designated follow-up period were documented, with a nonstandard number of visits and follow-up time across all study patients. Statistical analyses were conducted using SAS software (SAS Institute Inc), $\mathrm{R}$ software version 3.5.0 (R Foundation for Statistical Computing), and IBM SPSS Statistics software (IBM SPSS Statistics for Windows, Version 25).

\section{Results}

Patient Sample-A total of 25 ambiguous melanocytic neoplasms from 25 patients met the study inclusion criteria of a benign gene expression result with subsequent treatment as a benign neoplasm during follow-up. The 
patient sample statistics are summarized in Table 1. Most patients were younger than 65 years, with an average age at the time of biopsy of 48.4 years. All 25 neoplasms produced negative (benign) gene expression signature scores, all were diagnosed as benign nevi posttest by the submitting dermatopathologist, and all patients were initially treated in accordance with the benign diagnosis by the dermatologist(s) involved in clinical follow-up care. Prior to testing with the gene expression signature, most of these histopathologically indeterminate lesions received differential diagnoses, the most common of which were dysplastic nevus (84\%), melanoma arising from a nevus (72\%), and superficial spreading melanoma (64\%; eTable). After testing with the gene expression signature and receiving a benign score, most lesions received a single differential diagnosis of dysplastic nevus (88\%).

Follow-up and Survival-Clinical follow-up time ranged from 0.6 to 53.3 months, with a mean duration (SD)

\section{TABLE 1. Summary of Patient Demographics, Lesion Location, Test Results, and Interventions}

\begin{tabular}{|c|c|c|}
\hline Characteristic & Category/result & Total $(\mathrm{N}=25)$ \\
\hline \multirow[t]{2}{*}{ Gender, n (\%) } & Male & $13(52)$ \\
\hline & Female & $12(48)$ \\
\hline \multirow[t]{2}{*}{ Age at diagnosis, n (\%) } & $<65$ y & $19(76)$ \\
\hline & $\geq 65 y$ & $6(24)$ \\
\hline \multirow[t]{2}{*}{ Age at biopsy } & Mean (SL & $48.4(17.8) y$ \\
\hline & Med & $\begin{array}{l}51.0 \\
(33.0,64.0) y\end{array}$ \\
\hline \multirow{3}{*}{$\begin{array}{l}\text { Result of } 23 \text {-gene } \\
\text { expression signature, } \\
\mathrm{n}(\%)\end{array}$} & & $25(100)$ \\
\hline & Indeterminate & $0(0)$ \\
\hline & Malignant & $0(0)$ \\
\hline \multirow[t]{2}{*}{ Interventions, n (\%) } & Excision & $3(12)$ \\
\hline & $\begin{array}{l}\text { No surgical } \\
\text { intervention }\end{array}$ & $22(88)$ \\
\hline \multirow[t]{4}{*}{ Anatomic site, ${ }^{a} \mathrm{n}(\%)$} & Trunk & $18(72)$ \\
\hline & Arm & $4(16)$ \\
\hline & Lower extremity & $2(8)$ \\
\hline & Pelvis & $1(4)$ \\
\hline
\end{tabular}

Abbreviation: IQR, interquartile range.

aTrunk includes chest, back, and abdomen; arm includes shoulder; lower extremity includes foot. of 38.5 (16.6) months, and patients attended an average of 4 postbiopsy dermatology appointments (mean [SD], 4.6 [3.6]). According to the participating dermatology care providers, none of the 25 patients developed any indication during follow-up that the diagnosis of benign nevus was inaccurate. No patient had evidence of locally recurrent or metastatic melanoma, and none died during the study period.

Treatment/Interventions-The treatment recorded in the CRF was examination only for 21 of 25 patients, excision for 3 , and other for 1 (Table 2). Because the explanation for the selection of other in this case described an excision performed at the same anatomic location as the biopsy, this treatment also was considered an excision for purposes of the study analyses. The 3 excisions all occurred at the first postbiopsy dermatology encounter. Across all follow-up visits, no additional surgical interventions occurred (Table 2).

The first excision (case 1) involved a 67-year-old woman with a lesion on the mid pubic region described clinically as an atypical nevus that generated a pretest histopathologic differential diagnosis including dysplastic nevus, superficial spreading melanoma, and melanoma arising within a nevus (Table 3; Figure, A and B). The gene expression test result was benign (score, -5.4), and the final pathology report diagnosis was nevus with junctional dysplasia, moderate. Surgical excision was performed at the patient's first return visit, 505 days after initial diagnosis, with moderately dysplastic nevus as the recorded indication for removal. No repigmentation or other evidence of local recurrence or progression was detected, and the treating dermatologist indicated no suspicion that the original diagnosis of benign nevus was incorrect during the 23-month follow-up period.

TABLE 2. Follow-up Treatments/ Interventions in Case Report Forms

\begin{tabular}{lll} 
& $\begin{array}{l}\text { First visit, } \\
\mathrm{n}(\%)(\mathrm{N}=25)\end{array}$ & $\begin{array}{l}\text { Any visit, } \\
\mathrm{n}(\%)(\mathrm{N}=114)\end{array}$ \\
\hline Excision & $3^{\mathrm{a}}(12)$ & $3^{\mathrm{a}}(2.6)$ \\
\hline Excision + SLN biopsy & $0(0)$ & $0(0)$ \\
\hline Referral to surgeon & $0(0)$ & $0(0)$ \\
\hline $\begin{array}{l}\text { Other } \\
\text { Examination only }\end{array}$ & $1(4)$ & $15(13.2)$ \\
(no intervention) & $21(84)$ & $96(84.2)$ \\
\hline
\end{tabular}

Abbreviation: SLN, sentinel lymph node.

${ }^{a}$ Other was the recorded treatment for 1 case, but an excision at the same anatomic site as the biopsy was documented.

bOther was selected to document an appointment cancellation by the patient and a treatment unrelated to the tested lesion. 
The second excision (case 2) involved a 27-year-old woman with a pigmented neoplasm on the mid upper back (Figure, C and D) biopsied to rule out dysplastic nevus that resulted in a pretest histopathologic differential diagnosis of dysplastic nevus vs superficial spreading melanoma or melanoma arising within a nevus. The gene expression test result classified the lesion as benign (score, -2.9), and the final pathology diagnosis was nevus, compound, with moderate dysplasia. Despite the benign diagnosis, residual neoplasm (or pigmentation) at the biopsy site prompted the patient to request excision at her first postbiopsy visit, 22 days after testing (Table 3). The CRF completed by the dermatologist reported no indication that the benign diagnosis was inaccurate, but the patient was subsequently lost to follow-up.
The third excision (case 3) involved a 32-year-old woman with a pigmented lesion on the abdomen (Table 3; Figure, E and F). The clinical description was irregular-appearing black papule, nevus with atypia, and the histopathologic differential diagnosis again included dysplastic nevus, superficial spreading melanoma, and melanoma arising within a preexisting nevus. The gene expression signature result was benign (score, -7.2), and the final diagnosis issued within the accompanying pathology report was nevus with moderate junctional dysplasia. Despite the benign diagnosis, excision was performed 89 days after test result availability, with apparent residual pigmentation as the specified indication. As with the other 2 cases, the treating dermatologist confirmed
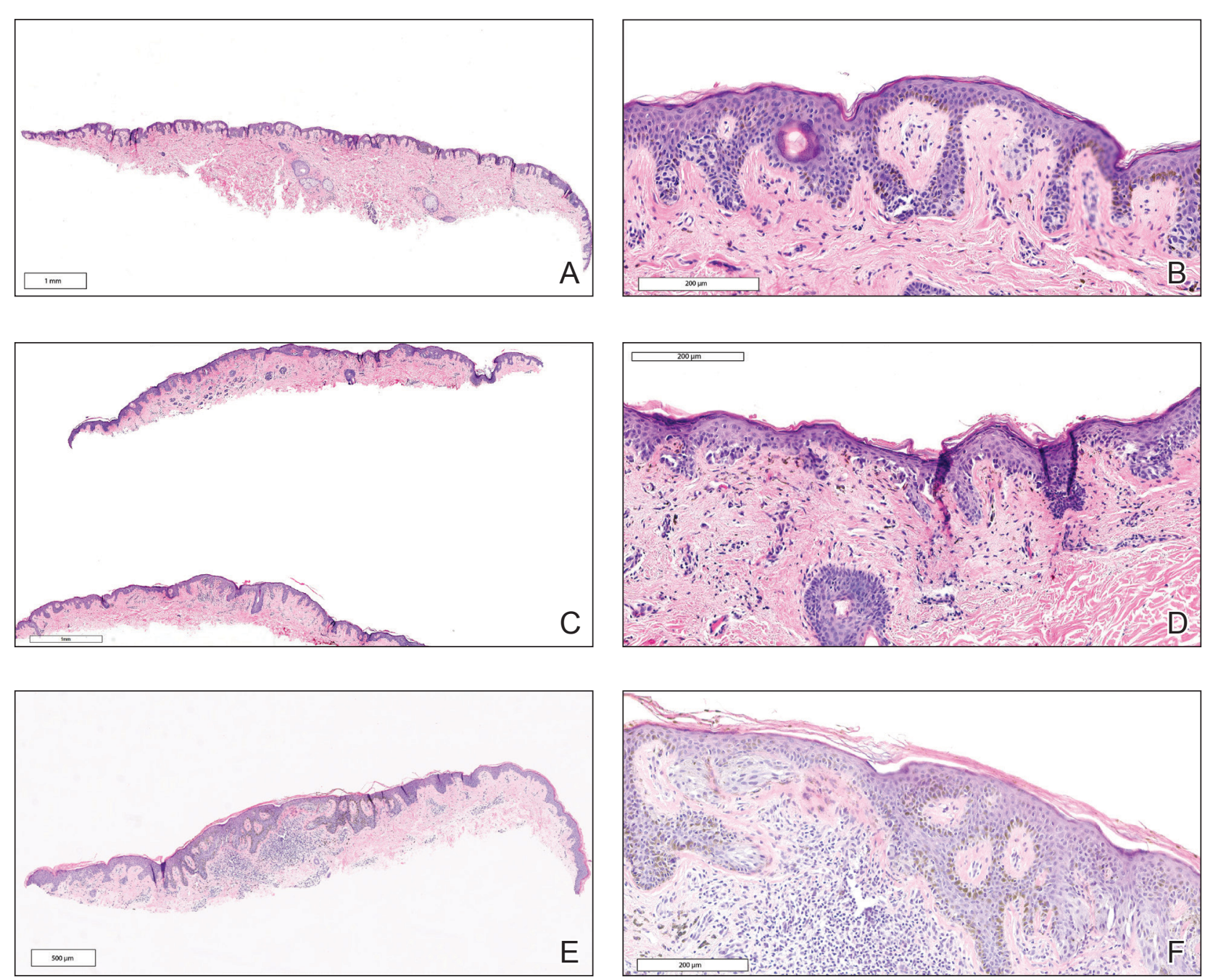

Histopathologic features of the 3 melanocytic neoplasms that were excised during the study follow-up period. The histopathologic differential diagnosis for each case included dysplastic nevus, superficial spreading melanoma, and melanoma arising within a preexisting nevus. All were classified as benign by the gene expression signature. For each, the indication for excision was patient or physician preference. $\mathrm{A}$ and $\mathrm{B}$, Case 1 (a 67-year-old woman with a lesion on the mid pubic region)(H\&E, original magnifications $\times 10$ and $\times 100)$. C and D, Case 2 (a 27 -year-old woman with a lesion on the back)(H\&E, original magnifications $\times 10$ and $\times 100)$. E and F, Case 3 (a 32-year-old woman with a lesion on the abdomen)(H\&E, original magnifications $\times 10$ and $\times 40)$. 
TABLE 3. Characteristics of Patients With Excised Lesions

\begin{tabular}{|c|c|c|c|c|c|c|c|}
\hline Case & Age, y & Gender & Anatomic site & Treatment & Indication & Follow-up, mo & Time to excision, $\mathrm{d}$ \\
\hline 1 & 67 & Female & Pelvis & Excision & $\begin{array}{l}\text { Patient/physician preference } \\
\text { (moderately dysplastic } \\
\text { nevus) }\end{array}$ & 23.1 & 505 \\
\hline 2 & 27 & Female & Back & Excision & $\begin{array}{l}\text { Patient/physician preference } \\
\text { (patient requested complete } \\
\text { removal) }\end{array}$ & 0.7 & 22 \\
\hline 3 & 32 & Female & Abdomen & Excision $^{a}$ & $\begin{array}{l}\text { Patient/physician preference } \\
\text { (residual lesion or } \\
\text { pigmentation) }\end{array}$ & 38.1 & 89 \\
\hline
\end{tabular}

${ }^{a}$ Actual treatment recorded was other, but free text documented an excision at the same anatomic site as the initial biopsy.

that neither clinical features nor follow-up events suggested malignancy.

\section{Comment}

This study followed a cohort of 25 patients with histopathologically ambiguous melanocytic neoplasms that were classified as benign by a diagnostic gene expression test with the intent of determining the outcomes of patients whose treatment aligned with their benign test result. All patients initially were managed according to their test result. During an average posttest clinical follow-up time of more than 3 years (38.5 months), the 25 biopsied lesions, most of which received a differential diagnosis of dysplastic nevus, were regarded as benign nevi by their dermatologists, and the vast majority $(88 \%)$ received no further surgical intervention. Three patients underwent subsequent excision of the biopsied lesion, with patient or physician preference as the indication in each instance. None of the 25 patients developed evidence of local recurrence, metastasis, or other findings that prompted doubt of the benign diagnosis. The absence of adverse events during clinical follow-up, particularly given that most lesions were not subjected to further intervention, supports use of the gene expression test as a safe and effective adjunct to the diagnosis and treatment of ambiguous melanocytic neoplasms by dermatologists and dermatopathologists.

Ambiguous melanocytic neoplasms evaluated without the aid of molecular adjuncts often result in equivocal or less-than-definitive diagnoses, and further surgical intervention is commonly undertaken to mitigate against the possibility of a missed melanoma. ${ }^{13}$ In this study, treatment that was aligned with the benign test result allowed most patients to avoid further surgical intervention, which suggests that adjunctive use of the gene signature can contribute to reductions in the physical and economic burdens imposed by unnecessary surgical interventions. ${ }^{15,16}$ Moreover, any means of increasing accurate and definitive diagnoses may produce an immediate impact on health outcomes by reducing the anxiety that uncertainty often provokes in patients and health care providers alike.

Study Limitations-This study must be interpreted within the context of its limitations. Obtaining meaningful patient outcome data is a common challenge in health care research due to the requisite length of follow-up and sometimes the lack of definitive evidence of adverse events. This is particularly difficult for melanocytic neoplasms because of an apparent inclination for patients with benign diagnoses to abandon follow-up and an increasing tendency for even minimal diagnostic uncertainty to prompt complete excision. Additionally, the only definitive clinical outcome for melanocytic neoplasms is distant metastasis, which (fortunately for patients) is relatively rare. Not surprisingly, studies documenting clinical outcomes of patients with ambiguous melanocytic neoplasms tested prospectively with diagnostic adjuncts are scarce, and this study's sample size and clinical follow-up compare favorably with the few that exist. ${ }^{17,18}$ Although most melanomas declare themselves through recurrence or metastasis within several years of initial biopsy, ${ }^{1,19}$ some are clinically dormant for as long as 10 years after initial detection. ${ }^{20,21}$ This may be particularly true for the small or early-stage lesions that now comprise the majority of biopsied neoplasms, and such events would go undetected by this study and many others. It also must be recognized that uneventful follow-up, regardless of duration, cannot prove that a biopsied melanocytic neoplasm was benign. Although only 5 patients had a followup time of less than 2 years (the time frame in which most recurrence or metastasis will occur), it cannot be definitively proven that a minimum of 2 years recurrenceor metastasis-free survival indicates a benign lesion. Many early-stage malignant melanomas are eradicated by complete excision or even by the initial biopsy if margins are uninvolved.

Because these limitations are intrinsic to melanocytic neoplasms and current management strategies, they 
pertain to all investigations seeking insights into biological potential through clinical outcomes. Similarly, all current diagnostic tools and procedures have the potential for sampling error, including histopathology. The rarity of adverse outcomes (recurrence and metastasis) in patients with benign test results within this cohort indicates that false-negative results are uncommon, which is further evidenced by a similar rarity of adverse events in prior studies of the gene expression signature..$^{8-10,22}$ A particular strength of this study is that most of the ambiguous melanocytic neoplasms followed did not undergo excision after the initial biopsy, an increasingly uncommon situation that may increase their likelihood to be informative.

It must be emphasized that the gene expression test, similar to other diagnostic adjuncts, is neither a replacement for histopathologic interpretation nor a substitute for judgment. As with all tests, it can produce falsepositive and false-negative results. Therefore, it should always be interpreted within the constellation of the many other data points that must be considered when making a distinction between benign nevus and malignant melanoma, including but not limited to patient age, family and personal history of melanoma, anatomic location, clinical features, and histopathologic findings. As is the case for many diseases, careful consideration of all relevant input is necessary to minimize the risk of misdiagnosis that might occur should any single data point prove inaccurate, including the results of adjunctive molecular tests.

\section{Conclusion}

Ancillary methods are emerging as useful tools for the diagnostic evaluation of melanocytic neoplasms that cannot be assigned definitive diagnoses using traditional techniques alone. This study suggests that patients with ambiguous melanocytic neoplasms may benefit from diagnoses and treatment decisions aligned with the results of a gene expression test, and that for those with a benign result, simple observation may be a safe alternative to surgical excision. This expands upon prior observations of the test's influence on diagnoses and treatment decisions and supports its role as part of dermatopathologists' and dermatologists' decision-making process for histopathologically ambiguous melanocytic lesions.

\section{REFERENCES}

1. Noone AM, Howlander N, Krapcho M, et al, eds. SEER Cancer Statistics Review, 1975-2015. National Cancer Institute website. Updated September 10, 2018. Accessed April 21, 2021 https://seer.cancer.gov/archive/csr/1975_2015/
2. Shoo BA, Sagebiel RW, Kashani-Sabet M. Discordance in the histopathologic diagnosis of melanoma at a melanoma referral center. J Am Acad Dermatol. 2010;62:751-756.

3. Veenhuizen KC, De Wit PE, Mooi WJ, et al. Quality assessment by expert opinion in melanoma pathology: experience of the pathology panel of the Dutch Melanoma Working Party. J Pathol. 1997; 182:266-272.

4. Elmore JG, Barnhill RL, Elder DE, et al. Pathologists' diagnosis of invasive melanoma and melanocytic proliferations: observer accuracy and reproducibility study. BMJ. 2017;357:j2813. doi:10.1136/bmj.j2813

5. Glusac EJ. The melanoma 'epidemic', a dermatopathologist's perspective. J Cutan Pathol. 2011;38:264-267.

6. Welch HG, Woloshin S, Schwartz LM. Skin biopsy rates and incidence of melanoma: population based ecological study. BMJ. 2005;331:481.

7. Swerlick RA, Chen S. The melanoma epidemic. Is increased surveillance the solution or the problem? Arch Dermatol, 1996;132:881-884.

8. Ko JS, Matharoo-Ball B, Billings SD, et al. Diagnostic distinction of malignant melanoma and benign nevi by a gene expression signature and correlation to clinical outcomes. Cancer Epidemiol Biomarkers Prev. 2017:26:1107-1113

9. Clarke LE, Flake DD 2nd, Busam K, et al. An independent validation of a gene expression signature to differentiate malignant melanoma from benign melanocytic nevi. Cancer, 2017;123:617-628.

10. Clarke LE, Warf BM, Flake DD 2nd, et al. Clinical validation of a gene expression signature that differentiates benign nevi from malignant melanoma. J Cutan Pathol. 2015;42:244-252.

11. Minca EC, Al-Rohil RN, Wang M, et al. Comparison between melanoma gene expression score and fluorescence in situ hybridization for the classification of melanocytic lesions. Mod Pathol. 2016;29:832-843.

12. Cockerell CJ, Tschen J, Evans B, et al. The influence of a gene expression signature on the diagnosis and recommended treatment of melanocytic tumors by dermatopathologists. Medicine (Baltimore). 2016;95:e4887. doi:10.1097/MD.0000000000004887

13. Cockerell C, Tschen J, Billings SD, et al. The influence of a geneexpression signature on the treatment of diagnostically challenging melanocytic lesions. Per Med. 2017;14:123-130.

14. Warf MB, Flake DD 2nd, Adams D, et al. Analytical validation of a melanoma diagnostic gene signature using formalin-fixed paraffinembedded melanocytic lesions. Biomark Med. 2015;9:407-416.

15. Guy GP Jr, Ekwueme DU, Tangka FK, et al. Melanoma treatment costs: a systematic review of the literature, 1990-2011. Am J Prev Med. 2012;43:537-545

16. Guy GP Jr, Machlin SR, Ekwueme DU, et al. Prevalence and costs of skin cancer treatment in the U.S., 2002-2006 and 2007-2011. Am J Prev Med. 2015;48:183-187.

17. Egnatios GL, Ferringer TC. Clinical follow-up of atypical spitzoid tumors analyzed by fluorescence in situ hybridization. Am J Dermatopathol. 2016;38:289-296.

18. Fischer AS, High WA. The difficulty in interpreting gene expression profiling in BAP-negative melanocytic tumors. J Cutan Pathol. 2018;45:659-666. doi:10.1111/cup.13277

19. Vollmer RT. The dynamics of death in melanoma. J Cutan Pathol. 2012;39:1075-1082.

20. Osella-Abate S, Ribero S, Sanlorenzo M, et al. Risk factors related to late metastases in 1,372 melanoma patients disease free more than 10 years. Int J Cancer. 2015;136:2453-2457.

21. Faries MB, Steen $S, Y e X$, et al. Late recurrence in melanoma: clinical implications of lost dormancy. J Am Coll Surg. 2013;217:27-34.

22. Ko JS, Clarke LE, Minca EC, et al. Correlation of melanoma gene expression score with clinical outcomes on a series of melanocytic lesions. Hum Pathol. 2019;86:213-221. 


\section{APPENDIX}

\section{eTABLE. Dermatopathologist Differential Diagnoses on Melanocytic Lesions Prior to Testing With the Gene Expression Signature $(\mathrm{N}=25)$}

\begin{tabular}{|c|c|c|}
\hline Differential diagnosis & Subtype ${ }^{a}$ & Patients, n (\%) \\
\hline \multirow[t]{3}{*}{ Pretest $^{\mathrm{b}}$ differential diagnosis 1} & Dysplastic nevus & $21(84)$ \\
\hline & Atypical junctional melanocytic proliferation & $3(12)$ \\
\hline & Acral nevus & $1(4)$ \\
\hline \multirow[t]{4}{*}{ Pretest $^{\mathrm{b}}$ differential diagnosis 2} & Melanoma arising from a nevus & $18(72)$ \\
\hline & Superficial spreading melanoma & \\
\hline & Dysplastic nevus & $3(12)$ \\
\hline & Acral melanoma & $1(4)$ \\
\hline \multirow[t]{6}{*}{ Pretest $^{\mathrm{b}}$ differential diagnosis 3} & Superficial spreading melanoma & $16(64)$ \\
\hline & Melanoma, not otherwise specified & $2(8)$ \\
\hline & Melanoma arising within a nevus & $3(12)$ \\
\hline & Lentigo maligna melanoma & $1(4)$ \\
\hline & Melanoma with blue nevus features & $1(4)$ \\
\hline & None selected & $2(8)$ \\
\hline \multirow[t]{4}{*}{ Posttest $^{\mathrm{b}}$ differential diagnosis 1} & Dysplastic $r$ & $22(88)$ \\
\hline & Junctional "helix" & $1(4)$ \\
\hline & al hyperplasia & $1(4)$ \\
\hline & I melanocytic proliferation & $1(4)$ \\
\hline \multirow{3}{*}{ Posttest $^{\mathrm{b}}$ differential diagnosis 2} & Atypical junctional melanocytic proliferation & $1(4)$ \\
\hline & Dysplastic nevus & $1(4)$ \\
\hline & None selected & $23(92)$ \\
\hline
\end{tabular}

a Subtypes were provided as free text by the diagnosing dermatopathologist.

${ }^{b}$ Pretesting or posttesting with the gene expression signature. 